\title{
Flujo espiratorio máximo: caracterización en un estudio en población adulta chilena; resultados basales de la cohorte del Maule (MAUCO)
}

\author{
VIVIANA ORENA C.***, GONZALO VALDIVIA C.***** y CATTERINA FERRECCIO R.**,***
}

\author{
Peak expiratory flow rate: characterization study in Chilean adult population; \\ baseline results of the Maule cohort (MAUCO)
}

Introduction: Chronic respiratory (CRD) diseases show high prevalence in developing countries, rural and deprived populations. Peak expiratory flow rate (PEFR) is a functional measurement obtained through forced expiratory used for clinical and research purposes. We described PEFR in a rural setting in an ongoing cohort study (Maule Cohort-MAUCO).Material and Method: Cross-sectional design in 3,465 adults (40-74 years) with PEFR ATS standard records (Mini-Wright flowmeter) using Gregg and Nunn values. PEFR $\leq 80 \%$ predicted were considered decreased. Sex, age, educational level, physical activity and tobacco smoking were obtained. Body mass index (BMI) was calculated based on anthropometrical measurements. Main Results: Sample had 63.9\% of women; mean age of $55( \pm 9)$ years, schooling of $9( \pm 4)$ years. Overweight and obesity were $43.1 \%$ and $41.5 \%$. Physical inactivity was $81.5 \%$ and $29.4 \%$ were current smokers. PEFR mean value was 330 ( \pm 80$) \mathrm{L} / \mathrm{min}$ (women) and 460 ( \pm 119$) \mathrm{L} / \mathrm{min}$ (men): Decreased PEFR was 50.6\% ([48.9-52.3]) with significant differences by age, schooling, BMI and physical activity. Conclusions: High prevalence of decreased PEFR was observed: PEFR showed variations according to sex, age, schooling, BMI and physical activity. As other LatinAmerican studies show, low values were also highly prevalent, suggesting that Gregg \& Nunn overestimated PEFR values. These results suggests the convenience of studying local environmental factors.

Key words: Cross-Sectional studies; peak expiratory flow rate; chronic diseases; health surveys.

\section{Resumen}

Introducción: Las enfermedades respiratorias crónicas tienen alta prevalencia en países en desarrollo, en poblaciones rurales y deprivadas. El flujo espiratorio máximo (FEM) obtenido mediante espiración forzada tiene uso clínico y de investigación. Describimos valores de medición del FEM en la medición basal de un estudio de cohorte en curso (Cohorte del Maule-MAUCO). Material y Método: Diseño transversal en 3.465 adultos (40-74 años) con registros de FEM (ATS). (Flujómetro Mini-Wright), usando valores de Gregg y Nunn. Valores $<80 \%$ del predicho se consideraron disminuidos. Se obtuvo sexo, edad, nivel educacional, actividad fisica y tabaquismo; se calculó índice de masa corporal (IMC) usando mediciones antropométricas. Resultados: La muestra tuvo 63,9\% de mujeres; edad media de $55( \pm 9)$ años, escolaridad de $9( \pm 4)$ años; sobrepeso y obesidad fueron 43,1\% y 41,5\%: 81,5\% fueron inactivos y 29,4\% fumadores actuales. El valor medio de FEM fue $330( \pm 80) \mathrm{L} /$ min (mujeres) y 460 ( \pm 119$) \mathrm{L} / \mathrm{min}$ (hombres): el FEM disminuido alcanzó el 50,6\% ([48,9-52,3]) con diferencias según edad, educación, IMC y actividad física. Conclusiones: Se observó alta prevalencia de FEM disminuido con variaciones según sexo, edad, escolaridad, IMC e inactividad fisica. Como otros estudios latinoamericanos, los valores bajos también fueron altamente prevalentes, sugiriendo sobreestimación de valores predichos al usar valores de Gregg \& Nunn. Estos resultados sugieren la conveniencia de estudiar factores ambientales locales.

Palabras clave: Estudios transversales; flujo espiratorio máximo; enfermedades crónicas no transmisibles; encuestas poblacionales.

* Escuela de Kinesiología, Facultad de Ciencias. Universidad Mayor Sede Temuco. Temuco, Chile.

** Departamento de Salud Pública, Facultad de Medicina. Pontificia Universidad Católica de Chile. Santiago, Chile.

***Advanced Center for Chronic Diseases (ACCDiS). 


\section{Introducción}

Las enfermedades respiratorias crónicas (ERC's) afectan la calidad de vida de quienes las padecen, lo cual determina un importante impacto económico en la persona, familia y sistemas de salud. La enfermedad pulmonar obstructiva crónica (EPOC) y el asma bronquial (AB) son las ERC's más comunes y representan un desafío para la salud pública tanto en países desarrollados como en vías de desarrollo por su alta frecuencia $\mathrm{y}$ tendencia al aumento ${ }^{1}$. En poblaciones rurales y más deprivadas económicamente el problema es mayor, ya que la pobreza se encuentra vinculada a exposiciones a contaminantes ambientales intra y extra domiciliarios $\mathrm{y} / \mathrm{u}$ ocupacionales nocivos para la salud, y a una baja sensibilidad para reconocer signos y síntomas respiratorios. Esto último, junto a la limitada disponibilidad de recurso humano especializado y restricciones de financiamiento en nivel primario de atención de salud (APS), explican la baja frecuencia diagnóstica de las ERC's, sospechándose en consecuencia, prevalencias poblacionales subestimadas en torno a ellas ${ }^{2-4}$.

La limitación crónica del flujo aéreo (LCFA) es un trastorno funcional que acompaña a un grupo de enfermedades y condiciones que favorecen el desarrollo de obstrucción al flujo aéreo espiratorio por obstrucción bronquial y/o disminución de la fuerza de retracción elástica del parénquima pulmonar. El uso de este término es apropiado sólo para las primeras etapas de estudio de un paciente mientras se avanza a una mayor precisión diagnóstica ${ }^{5}$. Cuando se desea saber si un sujeto presenta LCFA se realizan pruebas de función pulmonar estandarizadas que permiten medir volúmenes, flujos y capacidades pulmonares, así como también estudiar la frecuencia y distribución de enfermedades respiratorias en la población. Estas pruebas no permiten establecer un diagnóstico etiológico, pero informan el modo en que la enfermedad o condiciones ambientales alteran la mecánica ventilatoria normal, por lo que son útiles para objetivar la gravedad del trastorno funcional ${ }^{6,7}$.

Actualmente, dos pruebas de función pulmonar son ampliamente utilizadas en APS para el tamizaje, diagnóstico y control de las ERC's: la espirometría, examen con elevada sensibilidad y especificidad diagnóstica ${ }^{6}$, y la flujometría, herramienta útil para efectuar control terapéutico de enfermedades crónicas obstructivas y como prueba de diagnóstico sólo ante ausencia o imposibilidad de realizar la espirometría ${ }^{8-11}$.

La flujometría proporciona el flujo espiratorio máximo (FEM) que se consigue al haber espirado el $75-80 \%$ de la capacidad pulmonar total (CPT) dentro de los primeros $100 \mathrm{~ms}$ de espiración forzada. El FEM se expresa en litros/minuto o como porcentaje de su valor de referencia o predicho, y refleja el estado de las vías aéreas de gran calibre. Es un parámetro aceptado como medida independiente de función pulmonar tanto en la clínica como en estudios epidemiológicos ${ }^{9}$.

Este estudio evaluará las características de una medida de función pulmonar de uso rutinario en medicina respiratoria, en su aplicación a nivel poblacional en sujetos adultos. Adicionalmente tendrá por objetivo describir la frecuencia de alteración funcional respiratoria en población general adulta y residente en una zona rural de Chile, mediante una medida de función pulmonar general utilizando el patrón de referencia propuesto por Gregg \& Nunn ${ }^{24}$. El propósito es contribuir a la generación de estrategias para la promoción de la salud respiratoria así como también para apoyar su prevención y control.

El FEM integra una serie de mediciones basales del estudio de la Cohorte MAUCO, una investigación poblacional en curso del Centro de Estudios Avanzados de Enfermedades Crónicas $(\mathrm{ACCDiS})^{12}$.

\section{Material y Método}

El estudio MAUCO es el principal proyecto de investigación del Centro Avanzado de Enfermedades Crónicas (ACCDiS), de la Universidad de Chile y de la Pontificia Universidad Católica de Chile, en el cual 10.000 adultos de 38 a 74 años, residentes en la comuna de Molina de la Región del Maule, se están reclutando y siguiendo prospectivamente por un período de 10 años. La población en estudio tiene a todos los habitantes de Molina entre 38 y 78 años como potenciales participantes, identificados a través de un censo familiar, y sus mediciones contemplan la obtención de registros basales y de seguimiento $\left(\mathrm{al} 2^{\circ}\right.$, $5^{\circ}$ y $7^{\circ}$ año) realizados en el hogar y en centros locales de salud. La información se obtiene mediante la aplicación de cuestionarios y realización de mediciones estandarizadas en base a exámenes físicos y pruebas de laboratorio con muestras biológicas que incluyen la conformación de un biobanco. El estudio actual se realizó con datos secundarios basales del estudio MAUCO, utilizando un diseño transversal. De 4.225 sujetos con registros de FEM en la base de datos original a la fecha de este reporte, $293(6,9 \%)$ fueron excluidos por no presentar registros de talla. De 
los 3.932 restantes, 467 (11,8\%) fueron excluidos por no satisfacer el criterio de calidad para FEM (diferencia $<20 \mathrm{~L} / \mathrm{min}$ entre los dos registros más altos de FEM $)^{8}$. La mayor proporción de sujetos excluidos fueron mujeres, menores de 55 años y con 8 a 12 años de escolaridad. Finalmente, la muestra estuvo compuesta por los primeros 3.465 registros de adultos mujeres y hombres, reclutados entre los años 2014-2016, con edades entre 40-74 años e información disponible sobre edad, sexo y talla, variables indispensables para el cálculo del valor predicho del FEM en base a las ecuaciones corregidas de Gregg y Nunn $(2004)^{24}$. La medición del FEM se realizó con flujómetro Mini-Wright conforme a normativa estandarizada (ATS, 2005) (3) $^{13}$ El porcentaje de FEM observado respecto al predicho se clasificó según categorías de autocontrol del asma (Perelló et al., $2002)^{9}$. Se consideró FEM normal valores iguales o mayores a $81 \%$, deterioro leve entre el $65-80 \%$, deterioro moderado entre el $50-64 \%$ y deterioro severo valores iguales o menores a $49 \%$ tanto en mujeres como en hombres. Los antecedentes de sexo, edad, nivel educacional (NEDU en años de escolaridad), nivel de actividad física en tiempo libre, y hábito tabáquico (nunca fumador, exfumador y fumador actual) fueron obtenidos mediante encuesta. A través de mediciones físicas, se obtuvieron registros de peso $(\mathrm{kg})$ y talla $(\mathrm{m})$ que se utilizaron para el cálculo del índice de masa corporal (IMC en $\mathrm{kg} / \mathrm{m}^{2}$ ) que, posteriormente, fue clasificado en categorías de estado nutricional. El análisis se realizó con el programa SPSS (versión 21.0) y consideró la construcción de tablas de contingencia para el cálculo de frecuencias y la aplicación del test $\chi^{2}$ para asociación de variables, considerando como estadísticamente significativo un valor $\mathrm{p}<0,05$. El estudio contó con aprobación del Comité Ético-Científico de la Comisión de Investigación de la Pontificia Universidad Católica de Chile.

\section{Resultados}

Las características sociodemográficas de la muestra se presentan en la Tabla 1. De 3.465 sujetos, el $63,9 \%$ fueron mujeres. Para el total de la muestra, el promedio de edad fue de 55 años (DS \pm 9 ), la mayor proporción de sujetos se encontró entre los 50-54 años de edad y el promedio de años de escolaridad fue de 9 años (DS \pm 4 ), variables para las cuales no se observaron diferencias significativas por sexo. El 54,4\% tuvo un nivel educacional entre 8-12 años de escolaridad, el promedio de IMC fue de $30,0 \mathrm{~kg} /$ $\mathrm{m}^{2}$ (DS $\pm 4,9$ ), la mayor proporción de sujetos tuvo un estado nutricional correspondiente a la categoría de sobrepeso (IMC $25-29,9 \mathrm{~kg} / \mathrm{m}^{2}$ ), el $81,5 \%$ refirió no practicar actividad física y el $41,8 \%$ se declaró como nunca fumador, existiendo diferencias estadísticamente significativas por sexo para todas estas variables. Por su parte, las mujeres registraron un $43,6 \%$ de obesidad tipo I, II y III y los hombres un $38,0 \%$ (valor $\mathrm{p}<0,001$ ) así como un $28,3 \%$ y $31,7 \%$ de tabaquismo actual respectivamente (valor $\mathrm{p}<0,001$ ).

El promedio de FEM para el total de la muestra fue de $377 \mathrm{~L} / \mathrm{min}$ (DS \pm 114 ), con $330 \mathrm{~L} / \mathrm{min}$ $(\mathrm{DS} \pm 80)$ en mujeres y $460 \mathrm{~L} / \mathrm{min}(\mathrm{DS} \pm 119)$ en hombres, lo que determinó diferencias estadísticamente significativas por sexo (valor $\mathrm{p}<0,001$ ). Se observó que el promedio de FEM disminuyó con la edad y se estimó una disminución promedio de $19 \mathrm{~L} / \mathrm{min}$ en mujeres y $28 \mathrm{~L} / \mathrm{min}$ en hombres por cada grupo de edad (Tabla 2).

Los valores promedio de FEM observado fueron inferiores al promedio predicho en la totalidad de grupos de edad y en ambos sexos (Figuras 1 y 2 ).

Un $30,0 \%$ de la muestra fue clasificada con deterioro leve del FEM, un 15,2\% deterioro moderado y un $5,4 \%$ deterioro severo, lo que acumuló una prevalencia de FEM deteriorado de un $50,6 \%$ para el total de la muestra, utilizando el criterio de Perelló et al. ${ }^{9}$ para sujetos asmáticos en control. Las mujeres presentaron una menor prevalencia de FEM deteriorado que los hombres en todas sus categorías con un $29,4 \%$ de deterioro leve, un $13,2 \%$ de deterioro moderado y un $4,2 \%$ de deterioro severo, a diferencia de los hombres, quienes registraron un $31,1 \%$ de deterioro leve, $18,9 \%$ de deterioro moderado y $7,6 \%$ de deterioro severo. Lo anterior determinó diferencias significativas según sexo en las categorías de deterioro moderado y severo del FEM (valor $\mathrm{p}<0,001$ ) (Tabla 3).

Se observaron mayores prevalencias de FEM deteriorado en sujetos con NEDU menor a 8 años de escolaridad, a excepción de los hombres con deterioro leve del FEM en quienes la mayor prevalencia estuvo en aquellos con más de 12 años de escolaridad, lo cual determinó diferencias estadísticamente significativas por sexo (valor $\mathrm{p}<0,001$ ).

Al estudiar el FEM según estado nutricional y frecuencia de realización de actividad física en tiempo libre, las mayores prevalencias de deterioro moderado y severo estuvieron en sujetos de ambos sexos con algún grado de obesidad (tipo I, II o III) e inactivos físicamente en tiempo libre, con diferencias significativas según sexo (valor $\mathrm{p}<0,001)$. 
Tabla 1. Características sociodemográficas, antropométricas y distribución de factores conductuales en la muestra según sexo

\begin{tabular}{|c|c|c|c|c|}
\hline & $\begin{array}{c}\text { Mujeres } \\
\mathrm{n}=\mathbf{2 . 2 1 3}\end{array}$ & $\begin{array}{l}\text { Hombres } \\
\mathrm{n}=1.252\end{array}$ & $\begin{array}{c}\text { Total } \\
\mathrm{n}=\mathbf{3 . 4 6 5}\end{array}$ & Valor $\mathbf{p}$ \\
\hline Sexo n $(\%)$ & $2.213(63,9)$ & $1.252(36,1)$ & 3.465 & $0,000 * *$ \\
\hline Edad en años media ( \pm DS) & $55( \pm 9)$ & $55( \pm 9)$ & $55( \pm 9)$ & 0,82 \\
\hline \multicolumn{5}{|l|}{ Grupos de edad en años n (\%) } \\
\hline $40-44$ & $306(13,8)$ & $154(12,3)$ & $460(13,3)$ & 0,93 \\
\hline $45-49$ & $397(17,9)$ & $221(17,7)$ & $618(17,8)$ & \\
\hline $50-54$ & $453(20,5)$ & $261(20,8)$ & $714(20,6)$ & \\
\hline $55-59$ & $390(17,6)$ & $227(18,1)$ & $617(17,8)$ & \\
\hline $60-64$ & $279(12,6)$ & $166(13,3)$ & $445(12,8)$ & \\
\hline $65-69$ & $241(11,0)$ & $139(11,1)$ & $380(11,0)$ & \\
\hline $70-74$ & $147 \quad(6,6)$ & $84(6,7)$ & $231(6,7)$ & \\
\hline NEDU en años media ( \pm DS) & $8( \pm 4)$ & $9( \pm 4)$ & $9( \pm 4)$ & 0,50 \\
\hline \multicolumn{5}{|l|}{ Grupos de NEDU en años n (\%) } \\
\hline$<8$ & $831(37,9)$ & $413(33,2)$ & $1.244(36,2)$ & $0,03 *$ \\
\hline 8 y 12 & $1.162(52,9)$ & $709(57,0)$ & $1.871(54,4)$ & \\
\hline$>12$ & $202 \quad(9,2)$ & $121 \quad(9,7)$ & $323 \quad(9,4)$ & \\
\hline Peso en kg media ( \pm DS) & $71,3( \pm 12,9)$ & $81,1( \pm 14,1)$ & $74,8( \pm 14,2)$ & $0,000 * *$ \\
\hline Talla en m media $( \pm$ DS) & $1,54( \pm 0,1)$ & $1,67( \pm 0,1)$ & $1,59( \pm 0,1)$ & $0,000 * *$ \\
\hline IMC en $\mathrm{kg} / \mathrm{m}^{2}$ media $( \pm \mathrm{DS})$ & $30,0 \quad( \pm 5,2)$ & $29,1 \quad( \pm 4,4)$ & $30,0 \quad( \pm 4,9)$ & $0,000 * *$ \\
\hline \multicolumn{5}{|c|}{ Estado nutricional según IMC n (\%) } \\
\hline Normal $(<24,9)$ & $341(15,4)$ & $192(15,3)$ & $533(15,4)$ & $0,000 * *$ \\
\hline Sobrepeso $(25-29,9)$ & $909(41,1)$ & $584(46,7)$ & $1.493(43,1)$ & \\
\hline Obesidad tipo I $(30-34,9)$ & $623(28,2)$ & $375(30,0)$ & $998(28,8)$ & \\
\hline Obesidad tipo II $(35-39,9)$ & $241(10,9)$ & $81 \quad(6,5)$ & $322 \quad(9,3)$ & \\
\hline Obesidad tipo III $(>40)$ & $99 \quad(4,5)$ & $19 \quad(1,5)$ & $118 \quad(3,4)$ & \\
\hline \multicolumn{5}{|c|}{ Actividad física en tiempo libre n (\%) } \\
\hline$>3 \mathrm{v} / \mathrm{sem}$ & $188 \quad(8,7)$ & $103(8,4)$ & $291 \quad(8,5)$ & $0,000 * *$ \\
\hline $1-2 \mathrm{v} / \mathrm{sem}$ & $151 \quad(6,9)$ & $107 \quad(8,7)$ & $258 \quad(7,6)$ & \\
\hline$<4 \mathrm{v} / \mathrm{mes}$ & $30 \quad(1,4)$ & $51 \quad(4,2)$ & $81 \quad(2,4)$ & \\
\hline No practica & $1.804(83,0)$ & $965(78,7)$ & $2.769(81,5)$ & \\
\hline \multicolumn{5}{|l|}{ Hábito tabáquico n (\%) } \\
\hline Nunca fumador & $1.036(46,8)$ & $411 \quad(32,8)$ & $1.447(41,8)$ & $0,000 * *$ \\
\hline Ex-fumador & $552(24,9)$ & $445(35,5)$ & $997(28,8)$ & \\
\hline Fumador actual & $625(28,3)$ & $396(31,7)$ & $1.021 \quad(29,4)$ & \\
\hline
\end{tabular}

$\mathrm{n}=$ número de sujetos; $(\%)=$ porcentaje según columnas; DS = desviación estándar; NEDU = nivel educacional; $\mathrm{IMC}=$ índice de masa corporal; v/sem = veces por semana; $\mathrm{v} / \mathrm{mes}=$ veces por mes; ${ }^{*}$ valor $\mathrm{p}<0,05 ; * *$ valor $\mathrm{p}<0,001$. 
Tabla 2. Características del flujo espiratorio máximo (FEM) en la muestra según sexo

\begin{tabular}{|c|c|c|c|c|}
\hline & $\begin{array}{c}\text { Mujeres } \\
\mathrm{n}=\mathbf{2 . 2 1 3}\end{array}$ & $\begin{array}{l}\text { Hombres } \\
\mathrm{n}=\mathbf{1 . 2 5 2}\end{array}$ & $\begin{array}{c}\text { Total } \\
\mathrm{n}=\mathbf{3 . 4 6 5}\end{array}$ & Valor $\mathbf{p}$ \\
\hline Media en L/min ( $\pm \mathrm{DS})$ & $330( \pm 80)$ & $460( \pm 119)$ & $377( \pm 114)$ & $* * 0,000$ \\
\hline Rango en L/min (min-máx) & $70-560$ & $80-850$ & $70-850$ & \\
\hline \multicolumn{5}{|c|}{ Media de FEM según Grupos de Edad en L/min } \\
\hline 40-44 años Media ( \pm DS $)$ & $377( \pm 73)$ & $531( \pm 107)$ & $429( \pm 113)$ & \\
\hline 45-49 años Media ( \pm DS) & $355( \pm 76)$ & $492( \pm 114)$ & $404( \pm 113)$ & \\
\hline $50-54$ años $\quad$ Media $( \pm D S)$ & $340( \pm 70)$ & $471( \pm 116)$ & $389( \pm 110)$ & \\
\hline 55-59 años Media ( \pm DS) & $322( \pm 74)$ & $458( \pm 115)$ & $372( \pm 112)$ & \\
\hline 60-64 años $\quad$ Media $( \pm D S)$ & $310( \pm 71)$ & $428( \pm 108)$ & $354( \pm 104)$ & \\
\hline 65-69 años $\quad$ Media $( \pm D S)$ & $286( \pm 77)$ & $406( \pm 105)$ & $330( \pm 105)$ & \\
\hline 70-74 años $\quad$ Media ( \pm DS $)$ & $264( \pm 68)$ & $364( \pm 105)$ & $300( \pm 96)$ & \\
\hline
\end{tabular}

$\mathrm{L} / \mathrm{min}=$ litros por minuto; $\mathrm{DS}=$ desviación estándar; $* *$ valor $\mathrm{p}<0,001$.

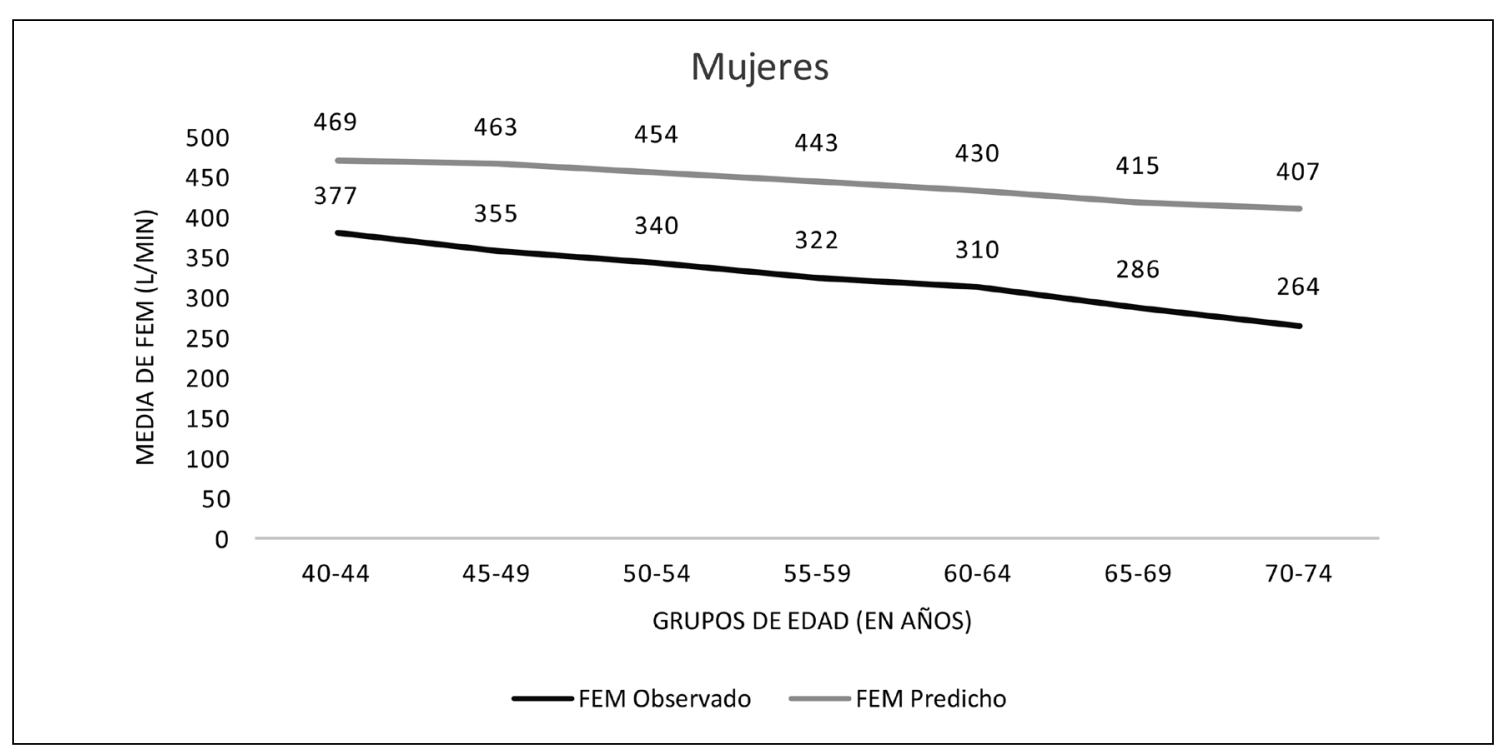

Figura 1. Promedio de FEM observado versus predicho en la muestra según grupos de edad, en mujeres.

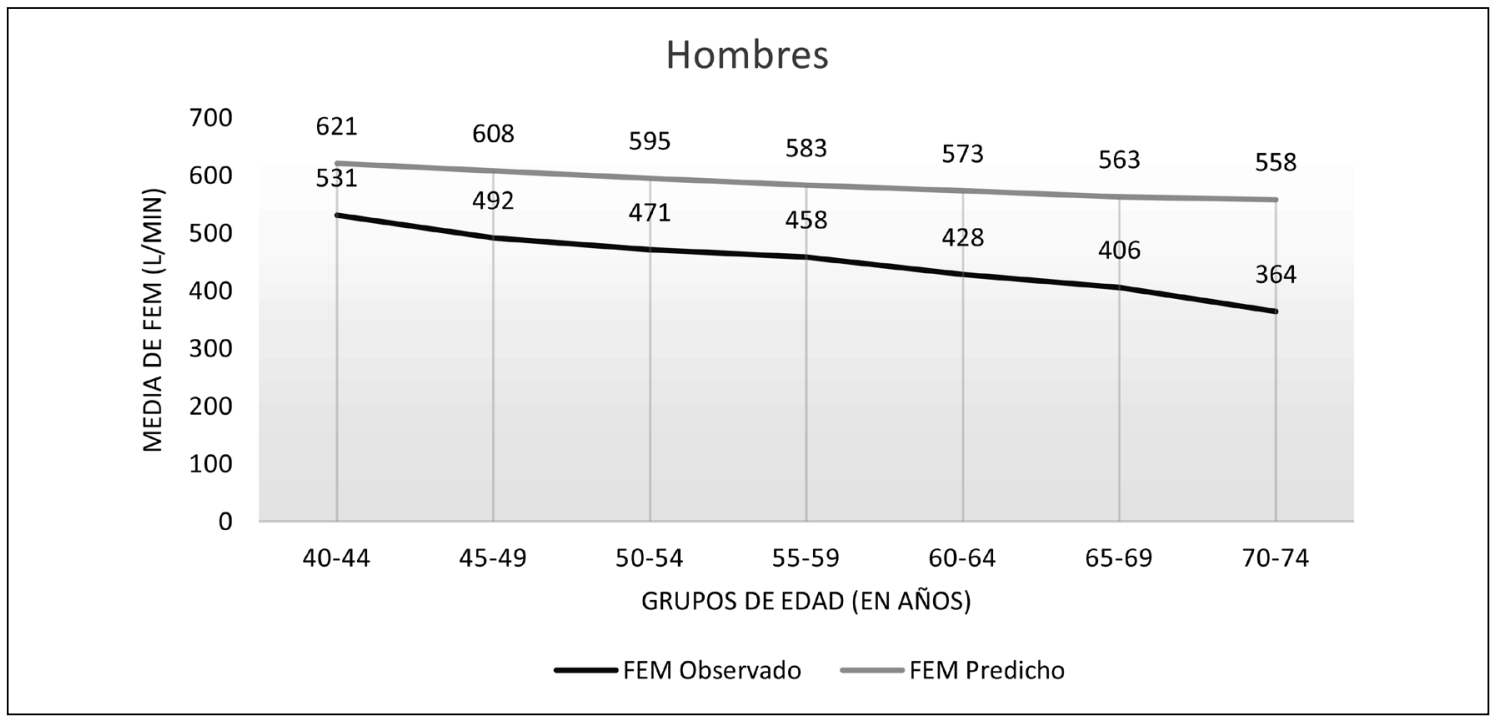

Figura 2. Promedio de FEM observado versus predicho en la muestra según grupos de edad, en hombres. 
Tabla 3. Prevalencia de FEM deteriorado en la muestra según sexo

\begin{tabular}{|llcccc|}
\hline & & $\begin{array}{c}\text { Mujeres } \\
\mathbf{n = 2 . 2 1 3}\end{array}$ & $\begin{array}{c}\text { Hombres } \\
\mathbf{n = 1 . 2 5 2}\end{array}$ & $\begin{array}{c}\text { Total } \\
\mathbf{n = 3 . 4 6 5}\end{array}$ & Valor p \\
Categorías de FEM n (\%) & Criterio & & & & \\
Normal & $\geq 81 \%$ & $1.178(53,2)$ & $531(42,4)$ & $1.709(49,3)$ & $0,000^{* *}$ \\
Deterioro leve & $65-80 \%$ & $651(29,4)$ & $389(31,1)$ & $1.040(30,0)$ & 0,29 \\
Deterioro moderado & $50-64 \%$ & $291(13,2)$ & $237(18,9)$ & $528(15,2)$ & $0,000 * *$ \\
Deterioro severo & $\leq 49 \%$ & $93(4,2)$ & $95(7,6)$ & $188(5,4)$ & $0,000 * *$ \\
\hline
\end{tabular}

Se presentan las categorías como porcentaje de FEM observado respecto al predicho; $n=$ número de sujetos; $(\%)=$ porcentaje según columna; FEM = flujo espiratorio máximo; **valor $\mathrm{p}<0,001$.

Finalmente, las mayores prevalencias de deterioro leve del FEM, estuvieron en: mujeres fumadoras actuales $(30,6 \%)$ y hombres nunca fumadores $(34,8 \%)$; de deterioro moderado en: mujeres nunca fumadoras $(14,3 \%)$ y hombres fumadores actuales $(19,9 \%)$, y de deterioro severo en: mujeres exfumadoras $(6,0 \%)$ y hombres nunca fumadores $(8,0 \%)$, lo que determinó diferencias significativas por sexo (valor $\mathrm{p}<0,001$ ).

\section{Discusión}

Las características sociodemográficas de la muestra manifiestan un predominio de nivel de escolaridad medio (entre 8-12 años), así como una pobre adherencia a estilos de vida saludables en términos de estado nutricional y frecuencia de realización de actividad física en tiempo libre. Estas características corresponden al histórico perfil epidemiológico descrito en poblaciones rurales y semirurales las cuales, en general, muestran conductas menos saludables que poblaciones urbanas $^{14-16}$.

En el estudio actual, se observó una prevalencia de tabaquismo superior a la informada por la Encuesta Nacional de Salud 2009-2010 ${ }^{17}$ para población rural, y se identificó un mayor porcentaje de mujeres fumadoras actuales (diarias + ocasionales) que hombres. Sin embargo, las características sociodemográficas de los participantes de la ENS no son del todo comparables con las de nuestro estudio: menor proporción de mujeres, rango de edad más amplio (15 años y más) y mayor nivel educacional.

Los promedios de FEM observado en nuestra muestra, tanto en mujeres como en hombres, fueron inferiores a los reportados por otros estudios realizados en población holandesa $(1994)^{18}$, norteamericana $(2001)^{19}$ y brasileña (2013 y $2016)^{20,21}$, los cuales también utilizaron la medi- ción del FEM con flujómetro Mini-Wright como principal variable de estudio; sin embargo, éstos estuvieron compuestos por muestras más jóvenes y residentes en zonas urbanas.

Por su parte, el promedio de FEM observado fue inferior al valor predicho en ambos sexos y en la totalidad de grupos de edad. Una posible explicación a este resultado podría ser la existencia de diversos factores que pueden afectar el FEM en las poblaciones: sexo, edad y la talla son los más reconocidos. Los hombres presentan mayores valores de FEM que las mujeres, al igual que los adultos jóvenes comparados con aquellos de edades avanzadas y sujetos de mayor estatura $^{22-23}$

Hay otros factores que también pueden influir en el resultado del FEM como son las diferencias anatómicas y fisiológicas entre mujeres y hombres en términos de fuerza de los músculos espiratorios, longitud y calibre de las vías aéreas, obstrucción extratorácica de las vías aéreas, limitaciones externas a la expansión torácica o que afectan la función de los músculos respiratorios, la integridad del sistema neural, la pérdida del volumen pulmonar y el aumento de la fuerza de retracción elástica como mecanismo compensatorio en procesos restrictivos, la influencia hormonal, bajo nivel socioeconómico, factores ambientales, tabaquismo y el nivel de actividad física ${ }^{22-23}$. La medición del FEM es también influida por el ciclo circadiano, registrándose los valores más altos alrededor de las 16:00 horas y los menos en la madrugada ${ }^{9}$. En nuestros registros, no contamos con la hora de tal medición.

La no disponibilidad de valores de referencia o predichos y puntos de corte validados de FEM en población chilena impide la comparación de los resultados con dicha referencia.

Una vez obtenido el FEM es necesario contrastarlo con su valor predicho dependiente del sexo, edad y la talla del sujeto para obtener el porcen- 
taje que, posteriormente, será clasificado según puntos de corte establecidos para cada población. Los valores predichos suelen ser obtenidos mediante una tabla de valores, un normograma o a través de ecuaciones de regresión que se construyen en base a "sujetos normales" con similares características antropométricas ${ }^{22-23}$. Los valores predichos reportados por estudios previos en otras poblaciones han revelado diferencias sustanciales entre ellos por la diversidad de criterios utilizados para la definición de "sujetos normales", el rendimiento del flujómetro y los distintos métodos de análisis de datos derivados de las ecuaciones de regresión ${ }^{22}$. Las ecuaciones modificadas de Gregg y Nunn (2004) ${ }^{24,25}$ utilizadas en el estudio actual para establecer los valores de referencia en mujeres y hombres, fueron construidas en base a población inglesa, quienes presentan características antropométricas que difieren de la población chilena, principalmente en términos de estatura; esta diferencia constituiría una explicación plausible a la inferioridad sistemáticamente observada del promedio de FEM respecto al valor predicho en los grupos estudiados. Así ha sido reportado en algunas evaluaciones realizadas en población general en Cuba, Brasil, Colombia y Perú, cuyos resultados concuerdan con este reporte, tanto con aquellos estudios que usaron flujómetro Mini-Wright como otros dispositivos para realizar sus mediciones, apuntando a un fenómeno de sobreestimación de los valores predichos del FEM al utilizar el estándar de Greg \& Nunn ${ }^{26-29}$.

Nuestro estudio demostró una alta prevalencia de FEM deteriorado en la muestra $(50,6 \%)$ comparado con la determinada por Singh y cols. $(25,3 \%)$, utilizando el mismo punto de corte como límite inferior de normalidad (LIN), es decir, un FEM $\leq 80 \%$ del valor predicho. Sin embargo, este último estudio estuvo constituido por una muestra más joven (edades promedio: 38 años en hombres y 33 años en mujeres), nunca fumadora, residente en una localidad menos deprivada económicamente y utilizó otras ecuaciones de regresión para establecer valores predi$\operatorname{chos}^{30}$. La diferencia esencial radica en el efecto de sobreestimación de valores de referencia ya comentado.

No obstante lo anterior, la alta prevalencia estimada de FEM deteriorado en el estudio actual podría explicarse por la posible influencia de factores de riesgo en común, que estarían promoviendo el deterioro de la función pulmonar en esta población, como podrían ser la utilización de biomasa (leña o carbón) como principal material de combustión (junto a ventilación deficiente del hogar), y la contaminación propia de la actividad agrícola (principal fuente laboral de la zona), que junto a las frecuentes quemas de superficies $\mathrm{y}$ al modelo social propio de sectores rurales $\mathrm{y}$ semirurales, podrían estar provocando una alta exposición a agentes ambientales intra y extra domiciliarios y/o a agentes ocupacionales nocivos para la función respiratoria, ya que en general, las mayores exposiciones ambientales y ocupacionales (pesticidas) ocurren en países en desarrollo y en zonas rurales ${ }^{31-34}$. Estimamos poco probable la existencia de algún fenómeno de sesgo de seleccción en la muestra, considerando la naturaleza censal del estudio de base.

Las fortalezas técnicas del estudio estuvieron determinadas por el empleo de una medida de función pulmonar en que todas sus mediciones fueron realizadas con el mismo dispositivo (flujómetro Mini-Wright, con lecturas de 0-800 L/ min) concordante con los criterios propuestos por la American Thoracic Society (ATS, 2005) para medición de la función pulmonar, además, la ejecución de una técnica estandarizada, la existencia de personal capacitado y la aplicación de criterio de calidad en los registros de FEM previo a los análisis.

Las limitaciones del estudio son el empleo de una única medición basal de FEM con utilidad limitada, el no constituir la flujometría una prueba de primera elección para la valoración de la función pulmonar, y que al ser una prueba dependiente del esfuerzo y del aprendizaje de la técnica de realización por parte del sujeto, puede ser menos confiable de no mediar una adecuada estandarización de la prueba. Finalmente, no se efectuaron controles biológicos en los flujómetros Mini-Wright durante el tiempo de realización de las mediciones basales. Sin embargo, la integridad de estos dispositivos fue revisada cada tres meses, y fueron inhabilitados y reemplazados luego de un máximo de 1.000 determinaciones.

\section{Conclusión}

El estudio MAUCO proporciona una adecuada oportunidad para el estudio de enfermedades respiratorias y permitió la realización de este primer estudio epidemiológico que utilizó el FEM, una medida general de función pulmonar, para describir la frecuencia de alteración funcional respiratoria en población chilena. El estudio muestra alta prevalencia de FEM deteriorado en mujeres y hombres, conforme a ecuaciones modificadas de Gregg y Nunn. La medición del FEM muestra variaciones según sexo, edad, nivel educacional, 
índice de masa corporal, nivel de actividad física en tiempo libre y hábito tabáquico. La naturaleza transversal del estudio limita el análisis de la influencia del consumo de tabaco. Los resultados sugieren la conveniencia de estudiar factores propios de entornos rurales y de establecer valores predichos y puntos de corte para FEM validados en población chilena.

\section{Agradecimientos}

Trabajo realizado en base a información proporcionada por el Proyecto FONDAP $\mathrm{N}^{\mathrm{o}}$ 15130011 del Advanced Center for Chronic Diseases (ACCDiS).

\section{Bibliografía}

1.- HURD S, LENFANT C. COPD: good lung health is the key. Lancet 2005; 366: 1832.

2.- SMITH K, HUMPHREYS J, WILSON M. Addressing the health disadvantage of rural populations: How does epidemiological evidence inform rural health policies and research? Australian Journal Rural Health 2008; 16: 56-66.

3.- BEARD J, TOMASKA N, EARNEST A, SUMMERHAYES R, MORGAN G. Influence of socioeconomic and cultural factors on rural health. Australian Journal Rural Health 2009; 17: 10-5.

4.- AÏT-KHALED N, ENARSON D, BOUSQUET J. Chronic respiratory diseases in developing countries: the burden and strategies for prevention and management. Bull World Health Organization 2001; 79: 971-9.

5.- HOGG J. Pathophysiology of airflow limitation in chronic obstructive pulmonary disease. Lancet 2004; 364 : 709-21.

6.- ARAYA M, PERTUZÉ J, VALDIVIA G, BATISTA AM, PÉREZ R, FUENTES H, et al. Calidad de las espirometrías en un estudio epidemiológico de terreno: Factores determinantes de la necesidad de repetir el examen en el estudio Platino-Chile. Rev Chil Enferm Respir 2005; 21: $155-63$.

7.- GUTIÉRREZ M, BEROÍZA T, BORZONE G, CAVIEDES I, CÉSPEDES J, GUTIÉRREZ M, et al. Espirometría: Manual de Procedimientos. Sociedad Chilena de Enfermedades Respiratorias. Rev Chil Enferm Respir 2007; 23: 31-42.

8.- CÉSPEDES J, GUTIÉRREZ M, OYARZÚN M, Comisión de Función Pulmonar de Adultos, Sociedad Chilena de Enfermedades Respiratorias. Flujometría en la práctica de atención primaria. Rev Chil Enferm Respir 2010; 26: 47-8.

9.- PERELLÓ M, RODRÍGUEZ R, Grupo de Respiratorio de La Societat Balear de Medicina Familiar I Comu- nitaria. Medidor de peak flow: técnica de manejo y utilidad en Atención Primaria. MEDIFAM 2002; 12 : 206-13.

10.- MINISTERIO DE SALUD. Guía Clínica Asma bronquial moderada y grave en menores de 15 años. Minsal 2011.

11.- TIAN J, ZHOU Y, CUI J, WANG D, WANG X, HU G, et al. Peak expiratory flow as a screening tool to detect airflow obstruction in a primary health care setting. Int J Tuberc Lung Dis 2012; 16: 674-80.

12.- FERRECCIO C, ROA J, BAMBS C, VIVES A, CORVALÁN A, CORTÉS S, et al. Study protocol for the Maule Cohort (MAUCO) of chronic diseases, Chile 2014-2024. BMC Public Health 2016; 16: 122.

13.- MILLER M, HANKINSON J, BRUSASCO V, BURGOS F, CASABURI R, COATES A, et al. Series “ATS/ ERS Task Force: Standardisation of lung function testing': Standardisation of spirometry". Eur Respir J 2005; 26: 319-38.

14.- HARTLEY D. Rural Health Disparities, Population Health and Rural Culture. Am J Public Health 2004; 94 : 1675-78.

15.- EBERHARDT M, PAMUK E. The Importance of Place of Residence: Examining Health in Rural and Nonrural Areas. Am J Public Health 2004; 94: 1682-86.

16.- RIVA M, CURTIS S, GAUVIN L, FAGG J. Unravelling the extent of inequalities in health across urban and rural areas: Evidence from a national sample in England. Social Science \& Medicine 2009; 68: 654-63.

17.- MINISTERIO DE SALUD (MINSAL). Encuesta Nacional de Salud (ENS) Chile 2009-2010. Disponible en: http://www.minsal.gob.cl. (Consultada el 20 de diciembre de 2016).

18.- BOEZEN H, SCHOUTEN J, POSTMA D, RIJCKEN B. Distribution of peak expiratory flow variability by age, gender and smoking habits in a random population sample aged 20-70 yrs. Eur Respir J 1994; 7: 1814-20.

19.- KLEIN B, MOSS S, KLEIN R, CRUICKSHANKS K. Peak Expiratory Flow Rate: Relationship to Risk Variables and Mortality. Diabetes Care 2001; 24: 1967-71.

20.- GIANINIS H, ANTUNES B, PASSARELLI R, SOUZA H, GASTALDI A. Effects of dorsal and lateral decubitus on peak expiratory flow in healthy subjects. Brazilian J Physical Therapy 2013; 17: 435-41.

21.- ANTUNES B, SOUZA H, GIANINIS H, PASSRRELLI-AMARO R, TAMBASCIO J, GASTALDI A. Peak expiratory flow in healthy, young, non-active subjects in seated, supine, and prone postures. Physiotherapy Theory and Practice 2016; 32: 489-93.

22.- QUANJER P, LEBOWITZ M, GREGG I, MILLER M, PEDERSEN O. Peak expiratory flow: conclusions and recommendations of a Working Party of the European Respiratory Society. Eur Respir J 1997; 10: 2s-8s.

23.- DIKSHIT M, RAJE S, AGRAWAL M. Lung functions with spirometry: an indian perspective-I. Peak expiratory flow rates. Ind J Physiol Pharmacol 2005; 49: 8-18. 
24.- GREGG I, NUNN A. Peak expiratory flow in normal subjects. Brit Med J 1973; 3: 282-84.

25.- MILLER M. Peak expiratory flow meter scale changes: implications for patients and health professionals. Airways J1 2004; 2: 80-2.

26.- ABASCAL M, GRAU R, LA ROSA A. Valores normales de flujo expiratorio forzado en la población de Ranchuelo. Rev Cubana de Medicina 2001; 40: 24352.

27.- DIAS C, VARANDA B, JAMAMI M, AMORIM V, TAYS K. Comparison between PEF values obtained from a population sample in the city of São Carlos, Brazil, and reference values. J Brasileiro Pneumol 2009; 35: 151-6.

28.- ROJAS M, VALERO A, FRANKY M. Determinación de valores normales de flujo respiratorio máximo en un grupo poblacional de 16 a 30 años en la ciudad de Tunja, 2007. Medicina (Bogotá) 2011; 33: 38-46.

29.- VERA A. Flujo pico espiratorio en personas adultas saludables de 20 a 60 años de edad en un hospital de la ciudad de Lima, de junio a noviembre de 2014 [tesis de pregrado]. Lima-Perú: Universidad Weiner; 2014. 74 p.

30.- SINGH J, KARMEE N, TRIPATHY R. Prevalence and
Correlates of Low PEFR among non-smoking tribal adults of Kandhamal district in Odisha: a cross sectional field study. Natl J Research in Community Medicine 2016; 5: 268-74.

31.- FLORES C, SOLÍS M, FORTT A, VALDIVIA G. Sintomatología respiratoria y enfermedad pulmonar obstructiva crónica y su asociación a contaminación intradomiciliaria en el Área Metropolitana de Santiago: Estudio Platino. Rev Chil Enferm Respir 2010; 26: 7280.

32.- RENNERT W, PORRAS R, MUNIZ G. The effects of smokeless cookstoves on peak expiratory flow rates in rural Honduras. J Public Health (Oxford) 2015; 455-60. Doi:10.1093/pubmed/fdu087

33.- BRUCE N, POPE D, REHFUESS E, BALAKRISHNAN A, ADAIR-ROHANI H, DORA C. WHO indoor air quality guidelines on household fuel combustion: Strategy implications of new evidence on interventions and exposure risk functions. Atmospheric Environment 2015; 106: 451-7.

34.- KURT O, ZHANG J, PINKERTON K. Pulmonary Health Effects of Air Pollution. Curr Opinion Pulm Med 2016; 22: 138-43.
Correspondencia a:

Dr. Gonzalo Valdivia Cabrera

Departamento de Salud Pública

Facultad de Medicina

Pontificia Universidad Católica de Chile.

Diagonal Paraguay 362, piso 2, Santiago, Chile.

8330073 (código postal)

Email: valdivia@med.puc.cl 